ENFOQUES JURIDICOS

REVISTA MULTIDISCIPLINAR DEL CEDECS

ISSN 26832070

Número 03

enero-junio 2021
Artículo: "La cláusula de apertura constitucional y su evolución en México"

Lilián Galván Bautista

DOI: https://doi.org/10.25009/ej.v0i3.2562

\title{
La cláusula de apertura constitucional y su evolución en México
}

Recibido $1^{\circ}$ septiembre 2020-Aceptado 12 noviembre 2020

Lilián Galván Bautista*

Profesora Asociada de Universidad de Salamanca y Letrada del Ilustre Colegio de Abogados de Salamanca, España

liliang@usal.es

RESUMEN: Este estudio describe la trayectoria histórica de la cláusula de apertura constitucional que poco a poco se ha consolidado con la evolución del constitucionalismo. Se observa que la cláusula de apertura constitucional, en sus varias modalidades, conduce a alcanzar una proyección internacional de los derechos fundamentales, con la finalidad de reforzar desde la propia Constitución su reconocimiento, respeto, protección y garantía en los países que desean instituir su democracia con un Estado Social de Derecho. Como consecuencia, nos invita a reflexionar en qué punto del camino se encuentra la evolución de los derechos fundamentales.

Palabras clave: Constitución, derechos fundamentales, cláusula de apertura constitucional.
ABSTRACT: This study describes the historical trajectory of the constitutional opening clause that has gradually consolidated with the evolution of constitutionalism. It can be seen that the Constitutional opening clause, in its various forms, leads to an international projection of fundamental rights in order to reinforce from the Constitution itself their recognition, respect, protection an assurance in countries that wish to establish their democracy with a Social State of Law. As a consequence, it invites us to reflect on where the evolution of fundamental rights finds itself.

Keywords: Constitution, fundamental rights, constitutional opening clause.

\footnotetext{
* Licenciada en Derecho por la UNAM, Doctora en Derecho Constitucional por la Universidad de Salamanca.
} 
ENFOQUES JURIDICOS

REVISTA MULTIDISCIPINAR DEL CEDECS

ISSN 26832070
Artículo: "La cláusula de apertura constitucional y su evolución en México"

Lilián Galván Bautista

DOI: https://doi.org/10.25009/ej.v0i3.2562

Número 03

enero-junio 2021

SUMARIO: Introducción. 1. El origen de la cláusula de apertura constitucional. 2. La cláusula de apertura con rango supraconstitucional. 3. La cláusula de apertura con rango constitucional. 4. La cláusula de apertura con rango supralegal. 5. El caso especial en la Constitución de México en la mutación de su cláusula de apertura.

\section{Introducción}

Realizando un análisis del concepto de la cláusula de apertura constitucional, a través de su historia del Derecho Constitucional y de su Teoría, observaremos su evolución e importancia en el Derecho Constitucional y cómo se produce su impacto en la Constitución Mexicana de 1917.

A nivel global el Derecho Constitucional ha adquirido actualmente gran importancia por el reconocimiento y protección que aporta a los derechos fundamentales, desde su origen en el Siglo XVIII con la Declaración de Independencia de los Estados de América en 1976 hasta la creación de los Tratados Internacionales sobre Derechos Humanos pactados en la segunda mitad del siglo XX. Cuya consecuencia ha sido dar la posibilidad de establecer en Europa un Derecho Constitucional Internacional con la Unión Europea.

Con la cláusula de apertura constitucional se busca una interpretación de los derechos fundamentales que están reconocidos en la Constitución y también consolidar progresivamente los que ya están reconocidos, de esta forma, se ve ampliada su cobertura en una triple proyección (vertical, horizontal y social), tomando como referencia la Teoría generacional de derechos humanos.

Por tanto, una de las funciones de la cláusula de apertura constitucional es vincular los Tratados Internacionales sobre Derechos Humanos con los derechos fundamentales que se reconocen dentro de la Constitución. Y esta vinculación puede adquirir tres tipos de nivel jerárquico que estará condicionada por la obligación que adquiera el Estado para salvaguardar los derechos fundamentales, tal como lo haya dispuesto el Poder Constituyente y en algunos casos particulares el Interprete de la Constitución. Nos referimos a las constituciones monistas internacionalistas, monistas nacionalistas y dualistas.

En la práctica, las formalidades oficiales que cada Estado establece para adherirse a un Tratado Internacional de Derechos Humanos, puede adquirir cuatro tipos de rango o valor jurídico que son: rango supraconstitucional, rango constitucional, rango supralegal y rango legal. La función es que mientras mayor jerarquía se le reconozca a estos Tratados en el orden interno, los requisitos de formalidad serán más estrictos.

Las constituciones que contienen una cláusula de apertura con rango supraconstitucional adquieren la capacidad de modificar su norma fundamental mediante la aceptación expresa del Tratado internacional y también se le considera ley constitucional. Pues, estos Tratados internacionales reconocen el sentido más amplio del derecho, por lo que deben tener preeminencia sobre la norma interna en materia de derechos 
ENFOQUES JURÍDICOS

REVISTA MULTIDISCIPLINAR DEL CEDECS

ISSN 26832070
Artículo: "La cláusula de apertura constitucional y su evolución en México"

Lilián Galván Bautista

Número 03

enero-junio 2021

DOI: https://doi.org/10.25009/ej.v0i3.2562

fundamentales, y los jueces no pueden apartarse de sus preceptos, al interpretar esta fuente, puesto que se les da una categoría superior al texto constitucional.

En el caso de la cláusula de apertura con rango constitucional adquieren la misma supremacía y rigidez los Tratados Internacionales de Derechos Humanos y la Constitución, para contribuir como fuentes de los derechos fundamentales reconocidos en ese Estado.

Sin embargo, el criterio jurisprudencial en algunas ocasiones ha asignado un reconocimiento superior a determinados derechos fundamentales al momento de confrontar la interpretación del Tratado Internacional de Derechos Humanos ante la Constitución y para otorgar una mayor garantía de dicho derecho, como ha sucedido en la Sentencia 2313-95 de Costa Rica.

La cláusula de apertura con rango supralegal se incorpora en las constituciones que otorgan a los Tratados Internacionales de Derechos Humanos un valor superior sobre las leyes nacionales. Así se les otorga la potestad de modificar o derogar las leyes que sean contrarias a los principios establecidos en los mismos, con independencia de que se trate de leyes anteriores o posteriores al Tratado.

Al estudiar el caso particular de la Constitución de México que entró en vigor en 1917, debemos tener en cuenta que los Tratados Internacionales de Derechos Humanos fueron posteriores a la misma, razón por la cual, originalmente no contenía esta cláusula de apertura constitucional. Por tanto, el artículo 133 al referirse a los Tratados Internacionales generalizaba su tratamiento, incluyendo a todos los que tuvieran un contenido de carácter político bilateral, multilateral ( $y$ donde se le dio cabida a los de materia de derechos humanos). Así todos los Tratados Internacionales adquirían la jerarquía de Ley Suprema, es decir, un rango legal. Por lo que se dificultó la reforma de ese precepto constitucional.

La Suprema Corte de Justicia de la Nación, interprete de la Constitución Mexicana, consideraba en su jurisprudencia que la redacción de la última parte del artículo 133 daba a los Tratados Internacionales un rango intermedio superior a la ley suprema, pero inferior a la Constitución, debido a su formalidad normativa, quedando dichos Tratados sin eficacia jurídica en los puntos que fuesen contrarios a la Constitución.

Sin embargo, en 1999 la interpretación aportada por la máxima autoridad judicial decidió elevar los Tratados Internacionales a un rango equitativo a la Constitución. Esto dio pie a que varios Jueces ampliaran dicha interpretación distinguiendo desde 2004 de entre los todos Tratados Internacionales aquellos que hacen referencia a los derechos humanos bajo el principio pro-homine y manifestando en 2010 que era necesaria una reforma constitucional sobre estos Tratados en vinculación a los artículos 1 y 15 de la Constitución Mexicana.

La reforma constitucional se produjo en junio de 2011, cambiando la redacción del segundo párrafo del artículo 1 al permitir "la protección más amplia" de los derechos fundamentales. De este modo, la transformación de la cláusula de apertura constitucional en México ha ido escalando todos los rangos iniciando desde el rango legal hasta el rango 
ENFOQUES JURIDICOS

REVISTA MULTIDISCIPLINAR DEL CEDEGS

ISSN 26832070
Artículo: "La cláusula de apertura constitucional y su evolución en México"

Lilián Galván Bautista

DOI: https://doi.org/10.25009/ej.v0i3.2562

Número 03

enero-junio 2021

constitucional y en algunas interpretaciones se considera que tiene un rango supraconstitucional. Por lo que su transformación continúa en proceso.

\section{El origen de la cláusula de apertura constitucional}

El constituyente ha confeccionado la cláusula de apertura constitucional, para que el catálogo de derechos y libertades, que se reconocen en la Constitución, incluya o amplíe su contenido conforme a los derechos humanos que han sido reconocidos en los Tratados Internacionales que ha firmado cada país para adherirlos a su ordenamiento.

El primer dato histórico en las constituciones escritas que hace una relación entre el ordenamiento internacional y el ordenamiento interno se manifestó por primera vez en la Constitución de los Estados Unidos de América de 1787 artículo VI.2, estableciendo que ambos sistemas jurídicos conformaran la Ley Suprema para este país.

Otro antecedente importante lo conforma la Constitución de Weimar de 1919, en donde reconoce el principio de origen anglosajón, el cual considera que la Ley Internacional es una parte de la Ley del Estado. Proclamando así en su artículo 4ㅇ la observancia de estas normas, que se consideran obligatorias para el derecho federal alemán.

Cabe recordar que la Constitución mexicana de 1957 fue inspirada en la citada Constitución Estadounidense, al manifestar en su artículo 126 que "esta Constitución, las Leyes del Congreso y de la Unión que emanen de ella y todos los Tratados hechos o que se hicieren por el Presidente de la República con aprobación del Congreso serán la Ley suprema de toda la Unión ...”, dicho precepto permanece vigente en la actual Constitución de 1917 con una ligera reforma.

Pero, la inclusión de los Tratados Internacionales de Derechos Humanos crea el mecanismo jurídico para combatir las violaciones a estos derechos. Por eso, estos Tratados tienen un efecto reparador y represivo sobre los obstáculos existentes para el cumplimiento de los derechos fundamentales (Fix, 1964: 77).

La cláusula de apertura constitucional posibilita que los ciudadanos acudan ante instancias internacionales o regionales encargadas de garantizar el orden constitucional interno en virtud, pues esta es la consecuencia de la adhesión del Estado. Se trata de procedimientos seguidos ante una jurisdicción internacional que protege los derechos y las libertades plasmadas en los documentos de carácter internacional aceptados por los Estados. En México establece un precedente con la Sentencia del Comisión Interamericana de Derechos Humanos en el caso Radilla Pacheco vs. Estados Unidos Mexicanos. (Montemayor, 2010:43 y sigs.). Por tal motivo, la cláusula de apertura constitucional puede servir, siempre que así se permita, para ampliar la configuración y protección interna de un derecho fundamental, cuando las mismas sean inferiores a lo preceptuado en el plano internacional, tal y como lo contempla el artículo 10.2 de la Constitución Española de 1978, misma que fue inspirada por la vigente Constitución Alemana. Esto permite que se 
ENFOQUES JURÍDICOS

REVISTA MULTIDISCIPLINAR DEL CEDECS

ISSN 26832070
Artículo: "La cláusula de apertura constitucional y su evolución en México"

Lilián Galván Bautista

Número 03

enero-junio 2021

DOI: https://doi.org/10.25009/ej.v0i3.2562

Introduzca una nueva garantía: las leyes que desarrollan el ejercicio de los derechos deberán respetar el contenido esencial de ese derecho (García, 1981: 143, 145).

Por lo tanto, el contenido de la cláusula de apertura constitucional conforma dos partes, una de las cuales, se constituye por las características constitucionales que varían en función del rango constitucional que se adapte a los intereses jurídicos y políticos del país que los establezca y, la otra parte, se integra por la dimensión que compone el contenido esencial de los derechos humanos. Siendo su función el deber de tutelar y hacer respetar el contenido esencial de los derechos fundamentales, plasmados tanto en la Constitución como en los Tratados y Acuerdos que cada país reconozca como parte de su ordenamiento jurídico.

Por tal motivo, para que estos derechos cumplan su función como tales, deben contener una triple proyección que se centra en tres deberes jurídicos reconocidos constitucionalmente que son, el deber de no lesionarlo (proyección vertical), el deber de protegerlo ante los demás individuos (proyección horizontal) y el deber de proporcionar los medios que garanticen el disfrute del derecho, (proyección social) (García, 1981: 75 - 76).

Esta triple proyección encuentra relación con la idea de "generaciones de derechos humanos", que se ha utilizado por juristas como Norberto Bobbio, Karel Vasak y Antonio E. Pérez Luño, al momento de clasificar la evolución histórica del reconocimiento de los derechos y libertades según el tipo de derecho de que se trate, atendiendo a una visión lineal o reduccionista del desarrollo histórico de los derechos humanos. Por tanto, nos encontramos en la primera generación los derechos de defensa, en la segunda generación, que comprende los derechos de contenido socioeconómico que implican obligaciones positivas para el Estado, y una tercera generación, la de aquellos derechos que responden a necesidades surgidas de las transformaciones tecnológicas de la sociedad posindustrial, tales como el derecho al medio ambiente, la protección de los consumidores y otros grupos sociales y la defensa frente al control de datos personales mediante la informática.

Se observa que la vinculación que hay con los Tratados Internacionales sobre Derechos Humanos en las constituciones de otros países se clasifican para efectos de nivel jerárquico que tienen los Tratados Internacionales en el derecho interno. Lo cual nos abre un panorama a la realidad jurídica a la que se enfrentan los derechos fundamentales, a fin de encontrar las diversas formas en las que se proyectan las cláusulas de apertura constitucional.

Aunque la tendencia en algunos países de Latinoamérica como ha sido el caso de Cuba y actualmente de Venezuela, es que cada vez sean inaplicables las normas sobre Tratados Internacionales de Derechos Humanos, el objetivo del Derecho Constitucional a través de la cláusula de apertura constitucional es que los Estados se obliguen a salvaguardar el derecho interno frente a posibles incompatibilidades de algún Tratado Internacional. Esto sucede cuando la consecuencia jurídica desemboca en un enfrentamiento entre derecho interno y derecho internacional, en el caso que la legislación interna, que se produce en un 
ENFOQUES JURÍDICOS

REVISTA MULTIDISCIPLINAR DEL CEDECS

ISSN 26832070
Artículo: "La cláusula de apertura constitucional y su evolución en México"

Lilián Galván Bautista

Número 03

enero-junio 2021

DOI: https://doi.org/10.25009/ej.v0i3.2562

país, adopte un sistema de jerarquización de normas de carácter nacionalista. (Corcuera, 2002: 156).

Las posibles vinculaciones que hay con los Tratados Internacionales sobre derechos humanos en las constituciones se han clasificado para efectos de nivel jerárquico, conocidas como: monistas internacionalistas, monistas nacionalistas y dualistas, de acuerdo a la terminología que utilizan (Arellano, 1999) y (Sepúlveda., 1973: 68 y 76). Por lo que, la realidad jurídica conduce a encontrar diversas formas en las que se proyecten los derechos humanos en sus constituciones desde la cláusula de apertura constitucional.

Entre las diversas formas observamos, que la corriente nacionalista coloca a los Tratados Internacionales en un nivel jerárquico inferior a la legislación interna; por ello, en caso de incompatibilidad entre las disposiciones de un Tratado y la legislación interna, prevalece esta última. En cambio, en la corriente internacionalista se coloca a los Tratados Internacionales en un nivel jerárquico superior a la legislación interna, por ello, en caso de incompatibilidad prevalece el Tratado internacional, como ocurre en la Constitución de España de 1978.

Por otra parte, la corriente dualista distingue los regímenes jurídicos y ámbitos de aplicación de los Tratados Internacionales de las leyes internas, lo que da como resultado que se tengan, conceptualmente, dos órdenes jurídicos separados. El régimen de derecho interno regula las relaciones jurídicas y sus consecuencias dentro de su jurisdicción, y los Tratados Internacionales regulan los compromisos entre el Estado correspondiente con las contrapartes del Tratado, sin que el mismo tenga efectos en el derecho de ese Estado.

En la práctica nos encontramos con una gran variedad de métodos utilizados para adoptar estos Tratados Internacionales en el derecho interno. Estos métodos pueden diferir dentro del mismo Estado, dependiendo de la naturaleza y los términos del Tratado y, a veces también, en la elección del Estado respecto a la manera de instrumentarlo (Gonzaini, 1995: 16). Pues, existen regímenes que permiten celebrar Tratados con la firma de quien tenga poderes plenos, para el efecto, sin necesidad de ratificación por el órgano legislativo para considerarse vinculados al Tratado. Y, habrá países cuyas constituciones exijan la ratificación del órgano legislativo para considerarse vinculados al Tratado y habrá diferentes requisitos de votación que se deberían cumplir para la aprobación respectiva por la parte del órgano legislativo.

Por tanto, el Poder Constituyente dispone de cuatro opciones para otorgar un rango o valor jurídico a los Instrumentos Internacionales, que se van a introducir en su Ordenamiento jurídico interno, al momento de reconocerlos como parte de su Constitución y que se clasifican en: rango supraconstitucional, rango constitucional, rango supralegal y rango legal. Para ilustrar estos regímenes jurídicos citaremos algunos ejemplos de constituciones que nos muestran el nivel jerárquico en el que colocan a los Tratados Internacionales sobre Derechos Humanos ante la propia Constitución, para entender que normalmente, mientras mayor jerarquía se le reconozca a los Tratados Internacionales, en 
ENFOQUES JURIDICOS

REVISTA MULTIDISCIPLINAR DEL CEDEGS

ISSN 26832070
Artículo: "La cláusula de apertura constitucional y su evolución en México"

Lilián Galván Bautista

Número 03

enero-junio 2021

DOI: https://doi.org/10.25009/ej.v0i3.2562

el Orden jurídico interno, mayores serán los requisitos de carácter formal que exigen las Constituciones respectivas.

\section{La cláusula de apertura con rango supraconstitucional}

En las constituciones que otorgan un rango supraconstitucional a los Tratados Internacionales de Derechos Humanos, tienen que prevalecer incluso frente a la Constitución del Estado. En este caso, los instrumentos internacionales que promueven y protegen los derechos humanos deberán tener una aceptación formal y eficaz por el Estado (Saiz, 1999: 50 y 51). Sin embargo, se encuentran muchos obstáculos al momento de ser aplicados por el Poder Judicial o del Intérprete de la Constitución al ponderar la norma interna y la norma internacional, por lo que es preciso que el valor sea apreciado positivamente por el sujeto, lo cual, depende de un acto estimativo del Estado.

En la práctica se entiende que la Constitución se reforma por conducto de un Tratado Internacional, el cual, adquiere el carácter de una ley constitucional con capacidad de modificar la Norma Fundamental con el consentimiento expreso del Poder Constituyente.

La Constitución de Guatemala establece en su artículo 46, el principio general de que (sólo) en materia de derechos humanos los Tratados y Convenios tienen preeminencia sobre el derecho interno. En este sentido se entiende que los derechos humanos son valores que tienen por objeto la convivencia armónica de sus ciudadanos, donde el orden jurídico positivo se subordina a ellos, y los jueces que lo interpretan encuentran una fuente modelo del cual, no pueden apartarse sin caer en arbitrariedad o injusticia (Gozaini, 1995: 17).

Este es un claro ejemplo en el que se ha interpretado que los Tratados Internacionales sobre Derechos Humanos están por encima del texto constitucional. Al utilizar la Constitución de Guatemala el término "derecho interno", sin distinción alguna, se comprende que incluye al derecho constitucional. El motivo que originó esta norma fue gracias al impacto que tuvo la opinión consultiva de la Corte Interamericana de Derechos Humanos OC-3/83 de 8 de septiembre de 1983, en la cual, la Corte estableció que un país no puede, aunque su Constitución se lo permita, aplicar la pena de muerte a delitos para los cuales no estaba contemplada anteriormente en su legislación interna (Ayala, 1961: 142).

Pero la Constitución de España de 1978, considerada como el ejemplo de la cláusula de apertura supraconstitucional, en esta situación, conforme a sus artículos 9.1 y 53.1 se exige como condición para la aprobación de un Tratado contrario a la Constitución, la previa revisión de esta; en virtud de que el régimen político Español se establece en una Monarquía Parlamentaria, constituida en un Estado Social y Democrático de Derecho, en cuyo caso, los órganos legitimados pueden requerir al intérprete de la Constitución que es Tribunal Constitucional, órgano independiente el Poder Judicial, para que declare si existe o no esa contradicción señalada en el artículo 95. Pue de este modo, el Constituyente ha 
ENFOQUES JURIDICOS

REVISTA MULTIDISCIPLINAR DEL CEDEGS

ISSN 26832070

Número 03

enero-junio 2021
Artículo: "La cláusula de apertura constitucional y su evolución en México"

Lilián Galván Bautista

DOI: https://doi.org/10.25009/ej.v0i3.2562

previsto garantizar que exista una armonía en los derechos fundamentales reconocidos en la propia Constitución y la integración de Tratados Internacionales.

Cabe destacar, que las disposiciones constitucionales sobre derechos humanos deben interpretarse de acuerdo con los instrumentos internacionales sobre la materia, lo que, sin significar una sumisión definitiva a los Tratados, implica cierto nivel jerárquico superior de los Tratados sobre la Constitución en la medida en que esta debe ser interpretada en congruencia con aquellos.

\section{La cláusula de apertura con rango constitucional}

Cuando se reconoce rango constitucional a los Tratados Internacionales de Derechos Humanos, se equiparán a la misma jerarquía normativa de la Constitución y de esta forma adquieren la supremacía y la rigidez de la propia Constitución. El criterio jurídico de estos Estados que optan por este rango constitucional al aceptar como positivos los derechos fundamentales nos lleva a sostener que deben ser realidades escritas en un catálogo que los identifique y reconozca. Lo que verdaderamente importa es el esfuerzo intersubjetivo que concibe la practicidad a partir de la comprensión social que los incorpora y remedia como valores de tránsito constante (Pérez, 1984: 162).

Este criterio es el que sigue la redacción de la Constitución Argentina resultante de la reforma de 1994, en la que decide dar a los Tratados en general una jerarquía superior a las leyes; a los Tratados y Declaraciones vigentes sobre derechos humanos, que enumera expresa y taxativamente el artículo 75 inciso 22, les otorga la jerarquía constitucional; así los Tratados sobre Derechos Humanos podrán gozar de la jerarquía constitucional en caso de que luego de ser aprobados por el Congreso se les imponga el voto de las dos terceras partes de la totalidad de los miembros de cada Cámara. Con lo cual, los Tratados sobre Derechos Humanos gozan de la misma jerarquía que la Constitución y solo pueden ser denunciados previo cumplimiento de un procedimiento agraviado, previsto en la misma.

Dentro de estos ejemplos, es un caso singular, el de la Constitución de Costa Rica, en la que se otorgó a los Tratados una jerarquía supralegal, pero la jurisprudencia le ha asignado una jerarquía constitucional. En su artículo 7 establece el rango superior de los Tratados sobre las Leyes, pero, se entiende inferior a la Constitución. No obstante, el aparente rango supralegal que se deriva para todos los Tratados en Costa Rica, la jurisprudencia de la Sala IV Constitucional, le ha reconocido a los Tratados referentes a derechos humanos un valor constitucional, pudiendo incluso resultar supraconstitucional en la medida en que otorguen mayores derechos o garantías a las personas (Sentencia 2313-95).

\section{La cláusula de apertura con rango supralegal}

Las Constituciones que contienen una cláusula de apertura con rango supralegal, colocan a las normas de derecho internacional en un valor superior a las normas de derecho interno, aunque no pueden modificar la Constitución, es decir, los Tratados prevalecen en este caso 
ENFOQUES JURIDICOS

REVISTA MULTIDISCIPINAR DEL CEDECS

ISSN 26832070
Artículo: "La cláusula de apertura constitucional y su evolución en México"

Lilián Galván Bautista

Número 03

enero-junio 2021

DOI: https://doi.org/10.25009/ej.v0i3.2562

sobre las leyes nacionales. Uno de los países Latinoamericanos que han acogido este sistema es El Salvador.

Esta Constitución determina en su precepto 144 que, en caso de conflicto entre el Tratado y la Ley, prevalecerá el Tratado y el artículo 145 se refiere a la ratificación de dichos Tratados. De este modo, se aclara expresamente el rango supralegal de los Tratados. Pues como observamos, los Tratados tienen una jerarquía superior a las leyes secundarias independientemente de que su vigencia sea anterior o posterior a la aceptación del Tratado. El carácter supralegal se puede apreciar en la condición que permite que el Tratado pueda derogar la ley secundaria anterior a este, entendiéndose que las leyes posteriores no podrán ser aprobadas cuando contradigan algún Tratado; y tampoco, las leyes secundarias posteriores al Tratado podrán derogar o modificar las disposiciones de un Tratado.

Desde esta perspectiva, se parte del principio de que todo derecho puede cobrar vigencia a partir de un hecho práctico, como la publicación oficial, o por la ratificación del Poder Ejecutivo de un Tratado aprobado en el seno del Poder Legislativo. Así lo prescribe el artículo 145 de la Constitución de El Salvador donde se aprecian los requisitos formales para celebrar los Tratados en El Salvador que son: las facultades de ratificar los Tratados Internacionales corresponden a la Asamblea Legislativa y la celebración de aquellos al órgano ejecutivo, el cual, es presidido por el Presidente de la República. La Asamblea puede denegar la ratificación o ratificar el instrumento con reservas por considerar inconstitucionales o inconvenientes las partes objetadas.

Estos preceptos constitucionales nos muestran los lazos entre los valores jurídicos presentes y los que aspiran a contraerse. Es decir, cuando el movimiento político y jurídico de una sociedad se organiza como protector de los derechos fundamentales, surge una complementación entre las esferas de la sociología y el derecho, a lo que denomina "el gran salto desde el statu quo hasta la esperanza" (Gozaini, 1995: 17).

\section{El caso especial en la Constitución de México en la mutación de su cláusula de apertura}

La Constitución Mexicana de 1917, ha tenido que mutar para sobrevivir a lo largo de muchas e importantes reformas. Y para integrar una cláusula de apertura constitucional no ha sido la excepción.

Pues la introducción de los Tratados Internacionales en el marco jurídico mexicano se hace de una forma genérica, ya que la Constitución de México no determinaba el contenido de dichos Tratados Internacionales. Este motivo, tiene su explicación en la época que se elaboró la Constitución Política de los Estados Unidos Mexicanos que fue en el año 1917, momento en el que todavía no estaban desarrollados los mecanismos de protección internacional sobre derechos humanos, a diferencia de la Constitución de España que fue aprobada en 1978.

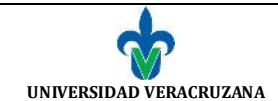

pp. $49-64$

드릅 
ENFOQUES JURIDICOS

REVISTA MULTIDISCIPLINAR DEL CEDECS

ISSN 26832070
Artículo: "La cláusula de apertura constitucional y su evolución en México"

Lilián Galván Bautista

DOI: https://doi.org/10.25009/ej.v0i3.2562

Número 03

enero-junio 2021

La norma fundamental que introduce a los Tratados Internacionales como parte del ordenamiento jurídico mexicano se desarrolla en el artículo 133 colocándolos en su primera redacción en la jerarquía normativa de la Ley suprema. Por lo que no se hacía ningún distingo en cuanto al contenido de estos Tratados Internacionales aceptados por el Estado Mexicano.

En términos generales las constituciones que otorgan a los Tratados Internacionales un rango legal tienen la misma jerarquía que la Ley interna, tal como en un inicio se contempló en la Constitución de México. Es el más difundido entre los distintos Estados de Latinoamérica, donde se proyecta una especial aceptación a los Tratados Internacionales sobre Derechos Humanos, "en este marco cada Estado supone una circunstancia individual para el progreso de los derechos fundamentales, lo cual, no impide que las normas internacionales orienten una definición propia, de imposible mutación por conveniencias particulares, ni limitaciones ostensibles a libertades plenamente reconocidas." (Goziani, 1995: 23 y 24).

En mayo de 1999, dio un cambio radical la interpretación del artículo 133 de la Constitución Mexicana, tras la Interpretación suscitada por la Suprema Corte de Justicia de la Nación, en su función de intérprete de la Constitución, el cual, se debatía entre dos cuestiones de interpretación (Galván, 2006: 646).

Del anterior criterio jurisprudencial destacaba el primer principio, considerar la supremacía constitucional, al interpretar la última parte del artículo 133 de la Constitución Mexicana, lo que se traduce en que los Tratados Internacionales ocupan un rango inmediato inferior a la Constitución, y cuando alguno de estos Tratados Internacionales, aun siendo aprobados por el Senado, contradiga o esté en oposición con los preceptos de la Constitución, en los puntos o actos en que esto acontezca, no debe tener eficacia jurídica.

El segundo principio, que la SCJN consideraba que los Tratados Internacionales por su formalidad normativa son actos materialmente legislativos por contener normas generales y abstractas, y debían ser considerados como leyes, teniendo como consecuencia que las autoridades competentes están obligadas a acatarlos y la sociedad y el Estado están interesados en su exacto cumplimiento. (Semanario Judicial de la Federación [SJF]; 7ạ época: 23; 163; 8a época: 236; 5a época: 43).

Sin embargo, en 1999 la SCJN cambió su criterio de interpretación, en una segunda Tesis Jurisprudencial referida al citado artículo 133 Constitucional, afirmando que los Tratados Internacionales se ubican jerárquicamente por encima de las Leyes Federales y en un segundo plano respecto de la Constitución Federal (Sentencia: 192,867 de 11 de mayo de 1999). Aquí valora los elementos inmersos en el sistema jurídico mexicano, ante el derecho internacional que son la jerarquía de los Tratados Internacionales, su aplicabilidad y la recepción de estos. Y, por tanto, cambia la ubicación de la cláusula de apertura constitucional de rango legal a un rango supralegal. 
ENFOQUES JURIDICOS

REVISTA MULTIDISCIPLINAR DEL CEDEGS

ISSN 26832070

Número 03

enero-junio 2021
Artículo: "La cláusula de apertura constitucional y su evolución en México"

Lilián Galván Bautista

DOI: https://doi.org/10.25009/ej.v0i3.2562

A raíz de esta Tesis Jurisprudencial, se torna complejo el sistema jurídico mexicano en cuanto al problema de su aplicación, si se tiene en cuenta que los sistemas normativos están articulados de tal manera que cuando hay una colisión entre Tratado Internacional y una norma de derecho interno debe aplicarse un criterio jerárquico.

Sin embargo, en un aspecto positivo, esta Jurisprudencia destaca porque sus argumentos se resumen en la idea que tanto el Tratado Internacional como la ley constitucional son normas de la unidad del Estado Federal y porque la interpretación del artículo 133 constitucional presenta una tesis que es armónica con diversos preceptos constitucionales y con la naturaleza que la propia Ley Federal señala a nuestro Estado Federal. (Carpizo, 2000: 75).

Con posterioridad a esta jurisprudencia se dictaron dos importantes tesis, una relativa al "principio pro homine" (Semanario Judicial de la Federación, [SJF] 2004: 2385) y otra titulada: "Tratados Internacionales. Su aplicación cuando amplían y reglamentan derechos fundamentales" (Semanario Judicial de la Federación, [SJF] 2004: 1896), ambas dan inicio al cambio de paradigma en la cláusula de apertura constitucional en México para reconocer, respetar, proteger y garantizar los derechos fundamentales que contiene esta Constitución al decidir que los Tratados Internacionales sobre Derechos Humanos tienen un rango paralelo a la Constitución, es decir, un rango constitucional.

Este nuevo sentido de interpretación de los derechos fundamentales a la luz de los Tratados Internacionales sobre derechos humanos no puede obviar el contenido de los artículos 1 y 15 de esta Norma Fundamental, preceptos que antes de la reforma de 2011 anteponían su garantía sin suspenderse, ni restringirse y establecía como principio la armonía entre los Tratados Internacionales de Derechos Humanos y la parte dogmática de la Constitución.

Por este motivo, se pronunció el Primer Tribunal Colegiado en materias administrativa y de trabajo del Décimo Primer circuito, al señalar en su Tesis Jurisprudencial 164.509 de mayo de 2010 que los Tratados o Convenciones suscritos por México relativos a derechos humanos deben ubicarse a nivel de la Constitución Política de los Estados Unidos Mexicanos.

Ante una variedad de interpretaciones sobre el rango que deben adoptar los Tratados Internacionales sobre Derechos Humanos en el ordenamiento mexicano se hace necesaria una reforma constitucional.

La mayoría de los Tratados Internacionales sobre Derechos Humanos forman parte del ordenamiento jurídico, en virtud de que México los ha ratificado. Sin embargo, el marco normativo internacional vinculante para México no siempre encuentra correspondencia con el derecho interno. Por lo que la tarea principal en materia de derechos humanos en México es armonizar ambos cuerpos legales a fin de garantizar una adecuada protección de valores fundamentales y evitar que incurra en responsabilidad internacional como consecuencia del incumplimiento de los Tratados Internacionales. Debe confeccionarse un 
ENFOQUES JURIDICOS

REVISTA MULTIDISCIPINAR DEL CEDECS

ISSN 26832070
Artículo: "La cláusula de apertura constitucional y su evolución en México"

Lilián Galván Bautista

Número 03

enero-junio 2021

DOI: https://doi.org/10.25009/ej.v0i3.2562

marco jurídico completo y eficaz para la protección y defensa de los derechos humanos. (Santos, 2004: 2).

Pero, el nuevo criterio de la SCJN no aporta una solución al problema que presenta la redacción del artículo 133 de la Constitución Mexicana para aplicar los Tratados Internacionales de Derechos Humanos (Corso, 2000: 46) por lo que no se descarta la necesidad de reformar el citado artículo 133 en concordancia con el artículo 1 constitucional. (Caballero, 2009: 283).

Ante la falta de actuación del Poder Legislativo, surgió una diversidad de criterios en el Poder Judicial intentando interpretar lo que quiso decir el Constituyente de México en el artículo 133; uno de estos criterios planteó la necesidad de introducir la cláusula de apertura constitucional en el sistema constitucional mexicano para integrar la protección y garantía que ofrece el derecho constitucional internacional compuesto por los Tratados Internacionales de Derechos Humanos.

Otro criterio que motivó la reforma constitucional en materia de Tratados Internacionales de Derechos Humanos, fuel el impacto que produjo el caso Radilla Pacheco vs Los Estados Unidos Mexicanos, al considerar que lo procedente es establecer el modo y alcance en que puede llevarse a cabo una operación que en diferentes grados ya se ha venido desarrollando, consistente en interpretar los derechos humanos reconocidos en el sistema jurídico mexicano observando el contenido de la jurisprudencia interamericana y de la Convención Americana de Derechos Humanos y otros Tratados Internacionales de conformidad con el lugar que ocupan en el entramado jurídico" (Cossío, 2014: 832).

Ante el reclamo por parte del Poder Judicial, la reforma constitucional, tan esperada, sobre el reconocimiento jerárquico de los Tratados Internacionales en materia de derechos humanos se proyectó en su artículo 1으, publicada el 10 de junio de 2011 . En concreto el párrafo segundo de este artículo es el que viene a definir la dimensión de la cláusula de apertura constitucional, que al permitir "la protección más amplia", con lo que termina colocando estos Tratados Internacionales en un rango supraconstitucional. Lo cual facilita "cierta flexibilización del rígido esquema ya clásico de distinción de esferas interior y exterior" en una relación de cooperación amistosa Estatal e Internacional. (Häberle, 2017: 283).

Los Tratados Internacionales a que se refiere la reforma del artículo 1 son aquellos celebrados por México conforme al artículo 133 en materia de derechos humanos. (Castañeda, 2015: 180). Así se establecen términos como "tarea común, cooperación y colaboración mutua términos clave propios del Estado cooperativo y su realidad exige los correspondientes esfuerzos" (Härbele, 2017: 285).

Finalmente, la cláusula de apertura constitucional en la Constitución Política de los Estados Unidos Mexicanos se ha configurado en su máxima dimensión adoptando el rango supraconstitucional, pero la tarea de aplicar los Tratados Internacionales de Derechos Humanos sobre de los derechos fundamentales de los mexicanos acaba de comenzar. 
ENFOQUES JURÍDICOS

REVISTA MULTIDISCIPLINAR DEL CEDECS

ISSN 26832070

Número 03

enero-junio 2021
Artículo: "La cláusula de apertura constitucional y su evolución en México"

Lilián Galván Bautista

DOI: https://doi.org/10.25009/ej.v0i3.2562

\section{Conclusiones}

En teoría el derecho constitucional mexicano ha conseguido solucionar la necesidad de dar una proyección interpretativa internacional a los derechos fundamentales que reconoce su Carta Magna, gracias a la doble labor legislativa y judicial que tienen los Tratados Internacionales sobre derechos humanos. Pero la labor no está terminada, plasmar los Tratados Internacionales en un rango supraconstitucional, es sólo la semilla que debe generar una mejor garantía de protección de todos los derechos fundamentales en México haciendo factible el pleno goce y disfrute de los mismos para avanzar a la democracia y a la estabilidad del Estado social de derecho.

En general, la cláusula de apertura constitucional en cualquiera de sus distintos rangos jerárquicos normativos tiene la función de garantizar, proteger, reconocer y respetar los derechos fundamentales en un país determinado con la colaboración de un Gobierno que debe tener la obligación de no interferir en el disfrute de los derechos fundamentales y de brindar prestaciones que posibiliten su desarrollo. De esta forma, la realidad jurídica en el ámbito constitucional interno tiene sus consecuencias en el ámbito constitucional internacional.

La importancia de la cláusula de apertura constitucional facilita que el sistema internacional o regional establezca la obligatoriedad de la ejecución de sus sentencias, cuando el Estado contradiga sus disposiciones constitucionales; y en tal caso deberá cumplir la obligación de adecuar su derecho constitucional al Convenio internacional.

Cuando el Poder Constituyente no ha introducido la cláusula de apertura constitucional, la solución que permite al ciudadano disfrutar los derechos fundamentales que se reconocen en los Tratados Internacionales, a los que este adherido el País en cuestión, debe surgir del Poder Judicial por tener la facultad para interpretar la Constitución y decidir mediante la jurisprudencia adecuar el derecho constitucional interno al derecho constitucional internacional como ocurrió en México y Costa Rica. 
ENFOQUES JURÍDICOS

REVISTA MULTIDISCIPLINAR DEL CEDEGS

ISSN 26832070

Número 03

enero-junio 2021
Artículo: "La cláusula de apertura constitucional y su evolución en México"

Lilián Galván Bautista

DOI: https://doi.org/10.25009/ej.v0i3.2562

\section{Fuentes de consulta}

\section{Libros de autor:}

Arellano García, C. (1999). "Primer curso de Derecho Internacional Público". México. Porrúa. 4a Edición.

Caballero Ochoa, J. L. (2009). "La incorporación de los Tratados Internacionales sobre derechos humanos en España y México". México. Porrúa.

Carpizo, J. (1984). "La Constitución de 1917, La formación del Estado Mexicano". México. Porrúa.

Castañeda, M. (2015). "Derecho Internacional de los Derechos Humano”. México. Publicado la edición de 25 Años, CNDH.

Corcuera Cavezut, S. (2002). "Derecho Constitucional y derecho internacional de los derechos humanos", México. Editorial Oxford, Colección de Textos Jurídicos Universitarios.

Fix Zamudio, H. (1964) "El Juicio de Amparo". México. Porrúa.

Galván Bautista, L. (2006). "Proyección Internacional de los derechos fundamentales. (Cláusula de apertura constitucional)". España. Ediciones Universidad de Salamanca.

Gozaíni, O. A. (1995). "El derecho procesal constitucional y los derechos humanos (vínculos y autonomía)". México. UNAM.

Häberle, P. (2017). “Pluralismo y Constitución. Estudios de Teoría Constitucional de la sociedad abierta". Madrid. Tecnos.

Montemayor, C. (2010) "Caso Radilla Pacheco vs. Estados Unidos Mexicanos". México, Estudios g\&q.

Perez Luño, A. (1984). "Derechos Humanos. Estado de Derecho y Constitución". Madrid. Tecnos. 
ENFOQUES JURIDICOS

REVISTA MULTIDISCIPLINAR DEL CEDECS

ISSN 26832070

Número 03

enero-junio 2021
Artículo: "La cláusula de apertura constitucional y su evolución en México"

Lilián Galván Bautista

DOI: https://doi.org/10.25009/ej.v0i3.2562

Saiz Arnaiz, A. (1999). "La apertura constitucional al Derecho Internacional y europeo de los derechos humanos. El artículo 10.2 de la Constitución Española". (Premio Rafael Martínez Emperador 1998). Madrid. Consejo General del Poder Judicial.

Sepulveda C. (1973). "Derecho Internacional”. México. Porrúa. 5ạ Edición.

\section{Capítulos de libro:}

Ayala Corao C. M. "La Jerarquización Constitucional de los Tratados", publicado en VV.AA. (1961). Acta de Federación de las Provincias Unidas de la Nueva Granada, Pensamiento Constitucional hispanoamericano hasta 1830. Caracas. Biblioteca de la Academia Nacional de la Historia.

Carpizo Mc Gregor, J. Publicado en VV.AA. (2002). "Comentarios a la Jurisprudencia Tratados Internacionales. Se ubica Jerárquicamente por encima de las Leyes Federales y en un Segundo Plano Respecto de la Constitución Federal". México. Instituto de Investigaciones Jurídicas de la UNAM.

Corso Sosa, E. Publicado en VV.AA. (2002). "Comentarios a la Jurisprudencia Tratados Internacionales. Se ubica Jerárquicamente por encima de las Leyes Federales y en un Segundo Plano Respecto de la Constitución Federal". México. Instituto de Investigaciones Jurídicas de la UNAM.

\section{Revistas:}

Santos, De Los M. A. (2004). "Derechos humanos: compromisos internacionales, obligaciones nacionales". México. Revista Mexicana de Justicia.

Cossío Díaz, J. R. (2014). "Algunas notas sobre el caso Rosendo Radilla Pacheco". México. Anuario Mexicano de Derecho Internacional, Vol. 14. Núm. C.

Legislación:

Constitución de la Nación de Argentina publicada en https://www.oas.org/dil/esp/Constitucion_de_la_Nacion_Argentina.pdf

Constitución de la República de Costa Rica publicada en http://www.eurosur.org/constituciones/co19-2.htm

Constitución de la República del Salvador publicada en https://www.oas.org/dil/esp/Constitucion_de_la_Republica_del_Salvador_1983.p df 
ENFOQUES JURIDICOS

REVISTA MULTIDISCIPLINAR DEL CEDECS

ISSN 26832070
Artículo: "La cláusula de apertura constitucional y su evolución en México"

Lilián Galván Bautista

DOI: https://doi.org/10.25009/ej.v0i3.2562

Número 03

enero-junio 2021

Constitución de los Estados Unidos de América de 1787. En National Archives, recuperado en https://www.archives.gov/espanol/constitucion.

Constitución de Weimar de 1919.1 Recuperado en https://ezequielsingman.files.wordpress.com/2016/03/constitucion-de-weimaralemania-19191.pdf

Constitución Española de 1978. Publicado en el BOE.es https://www.boe.es/legislacion/documentos/ConstitucionCASTELLANO.pdf

Constitución Política de la República de Guatemala publicada en https://www.cijc.org/es/NuestrasConstituciones/GUATEMALA-Constitucion.pdf

Constitución Política de los Estados Unidos Mexicanos publicada en http://www.diputados.gob.mx/LeyesBiblio/pdf_mov/Constitucion_Politica.pdf

\section{Sentencias:}

Jurisprudencia SCJN, Registro No. 164509, Localización: Novena Época, Instancia: Tribunales Colegiados de Circuito, Fuente: Semanario Judicial de la Federación y su Gaceta, XXXI, mayo de 2010, Página: 2079, Tesis: XI.1o.A.T.45 K, Tesis Aislada, Materia(s): Común.

Sentencias publicadas en el Semanario Judicial de la Federación; 1. - 7ạ época, Pleno, vols. 193-198, 1a Parte, pág. 163; 2.- 8a época, Tribunales colegiados de circuito, t. VI, 2aㅡ parte, 1, pág. 236. 3.- 7ạ época, sala, vol. 72, 3ạ parte, pág. 23; 5a época, pleno t. VI.

Tesis aislada, Materia(s): Administrativa, novena época, Instancia: Tribunales Colegiados de Circuito, Fuente: Semanario Judicial de la Federación y su Gaceta, t. XX, septiembre de 2004, Tesis: I.4o.A.440 A, Página: 1896. Amparo en revisión 99/2003. Ismael González Sánchez y otros. 21 de abril de 2004. Unanimidad de votos. Ponente: Hilario Bárcenas Chávez. Secretaria: Mariza Arellano Pompa.

Tesis aislada, Materia(s): Administrativa, novena época, Instancia: Tribunales Colegiados de Circuito, Fuente: Semanario Judicial de la Federación y su Gaceta, t. XX, octubre de 2004, Tesis: I.40.A.441 A, p. 2385. Amparo en revisión 799/2003. Ismael González Sánchez y otros. 21 de abril de 2004. Unanimidad de votos. Ponente: Hilario Bárcenas Chávez. Secretaria: Mariza Arellano Pompa.

Tesis Jurisprudencial 192.867, P. LXXVII/99 publicada en el Semanario Judicial de la Federación, pleno, t. X, México, 1999. 